\title{
Surface and edge states in topological semimetals
}

\author{
Rui-Lin Chu, Wen-Yu Shan, Jie Lu, and Shun-Qing Shen \\ Department of Physics and Center of Computational and Theoretical Physics, The University of Hong Kong, \\ Pokfulam Road, Hong Kong \\ (Received 22 September 2010; revised manuscript received 10 November 2010; published 15 February 2011)
}

\begin{abstract}
We study the topologically nontrivial semimetals by means of the six-band Kane model. Existence of surface states is explicitly demonstrated by calculating the local density of states (LDOS) on the material surface. In the strain-free condition, surface states are divided into two parts in the energy spectrum, one part is in the direct gap, the other part including the crossing point of surface state Dirac cone is submerged in the valence band. We also show how uniaxial strain induces an insulating band gap and raises the crossing point from the valence band into the band gap, making the system a true topological insulator. We predict the existence of helical edge states and spin Hall effect in the thin-film topological semimetals, which could be tested by future experiments. Disorder is found to significantly enhance the spin-Hall effect in the valence band of the thin films.
\end{abstract}

DOI: 10.1103/PhysRevB.83.075110

PACS number(s): 73.20.At, 73.61.At

\section{INTRODUCTION}

Topological insulators (TIs) have been recognized as novel states of quantum matter. ${ }^{1}$ They are of tremendous interest to both fundamental condensed matter physics and potential applications in spintronics as well as quantum computing. TIs are insulating in the bulk which is usually due to direct bulk band gaps. However, on the boundary there are topologically protected gapless surface or edge states. Usually in these materials the spin-orbital coupling is very strong such that the conduction band and valence band are inverted. Such phenomena have already been reported in the literature of the $1980 \mathrm{~s}^{2-6}$ However, the topological nature behind these phenomena was not revealed at the time. With the recent development of topological band theories, a series of materials including $\mathrm{HgTe}$ quantum well, $\mathrm{Bi}_{x} \mathrm{Sb}_{1-x}, \mathrm{Bi}_{2} \mathrm{Se}_{3}$, and $\mathrm{Bi}_{2} \mathrm{Te}_{3}$ have been theoretically predicted and experimentally realized as two-dimensional (2D) and three-dimensional (3D) TIs..$^{7-9,11-15}$ The search for TIs has been extended from these alloys and binary compounds to ternary compounds. Very recently a large family of materials, namely the Heusler-related and Li-based intermetallic ternary compounds, have been predicted to be promising 3D TIs through first principle calculations. ${ }^{16-22}$ The enormous variety in these compounds provides wide options for future material synthesizing of TIs. However, despite the fact that an insulating bulk is a critical prerequisite to the TI theory, none of these newly found materials are naturally band insulators. Many of them are semimetals or even metals. It has been shown that the band structure and band topology of many of these compounds closely resemble that of the zinc blende structure binary compound $\mathrm{HgTe}$ and CdTe. ${ }^{16-22}$

In this work we study the evolution of surface states in these topologically nontrivial compounds whose band structures closely resemble 3D HgTe by means of the six-band Kane model. By studying the local density of states (LDOS) on the material boundary, we demonstrate explicitly the existence of surface states in the direct band gap. Furthermore, we show that in the strain-free condition, the surface states are separated into two parts in the band structure. One part exists in the direct band gap, the other part of surface states (including the crossing point of the Dirac cone) submerges in the valence bands. The latter surface states have distinct momentumdependent spatial distribution from the former. By applying uniaxial strains, the crossing point can be raised up into the strain-induced insulating gap. In the thin films made of these materials, topologically protected helical edge states will emerge on the sample edges. In this way we show a crossover between 3D TIs and 2D TIs. In the strain-free condition, the bulk band gap can be controlled by tuning the film thickness. Meanwhile, we found that in the thin films, disorders or impurities can significantly enhance the spin-Hall effect (SHE) when the Fermi level is in the valence band.

\section{MODEL HAMILTONIAN}

3D HgTe and CdTe share the same zinc blende structure. The band topology of these two materials is distinguished by the band inversion at the $\Gamma$ point, which happens in $\mathrm{HgTe}$ but not CdTe. This causes HgTe to be topologically nontrivial while CdTe is trivial. ${ }^{3,11,28}$ The essential electronic properties of both are solely determined by the band structure near the Fermi surface at the $\Gamma$ point, where the bands possess $\Gamma_{6}$ ( $s$-type, doubly degenerate), $\Gamma_{8}$ ( $p$-type, $j=3 / 2$, quadruply degenerate), and $\Gamma_{7}$ ( $p$-type, $j=1 / 2$, doubly degenerate) symmetry. ${ }^{25}$ The band inversion in HgTe takes place because the $\Gamma_{6}$ bands appear below the $\Gamma_{8}$ band, whereas in the normal case (such as CdTe) $\Gamma_{6}$ is above $\Gamma_{8}$ (see Fig. 1). ${ }^{23,24}$ In this work, we use a six-band Kane model Hamiltonian which takes into account the $\Gamma_{6}$ and $\Gamma_{8}$ band. The spin-orbit split off $\Gamma_{7}$ band usually appears far below the $\Gamma_{6}$ and $\Gamma_{8}$ bands and hence can be neglected because it does not affect the low-energy approximation. The six-band Kane model describes the band structure near the $\Gamma$ point well and adequately captures the band inversion story. ${ }^{3,28}$ As expected, we find gapless surface states exist in the direct gap of 3D $\mathrm{HgTe}$ while absent in CdTe. 
The six-band Kane Hamiltonian is

$$
H_{0}=\left(\begin{array}{lccccc}
T & 0 & -\frac{1}{\sqrt{2}} P k_{+} & \sqrt{\frac{2}{3}} P k_{z} & \frac{1}{\sqrt{6}} P k_{-} & 0 \\
0 & T & 0 & -\frac{1}{\sqrt{6}} P k_{+} & \sqrt{\frac{2}{3}} P k_{z} & \frac{1}{\sqrt{2}} P k_{-} \\
-\frac{1}{\sqrt{2}} P k_{-} & 0 & U+V & 2 \sqrt{3} \bar{\gamma} B k_{-} k_{z} & \sqrt{3} \bar{\gamma} B k_{-}^{2} & 0 \\
\sqrt{\frac{2}{3}} P k_{z} & -\frac{1}{\sqrt{6}} P k_{-} & 2 \sqrt{3} \bar{\gamma} B k_{+} k_{z} & U-V & 0 & \sqrt{3} \bar{\gamma} B k_{-}^{2} \\
\frac{1}{\sqrt{6}} P k_{+} & \sqrt{\frac{2}{3}} P k_{z} & \sqrt{3} \bar{\gamma} B k_{+}^{2} & 0 & U-V & -2 \sqrt{3} \bar{\gamma} B k_{-} k_{z} \\
0 & \frac{1}{\sqrt{2}} P k_{+} & 0 & \sqrt{3} \bar{\gamma} B k_{+}^{2} & -2 \sqrt{3} \bar{\gamma} B k_{+} k_{z} & U+V
\end{array}\right),
$$

where

$$
\begin{gathered}
B=\frac{\hbar^{2}}{2 m_{0}}, \quad P=\sqrt{\frac{\hbar^{2} E_{p}}{2 m_{0}}}, \quad k_{\|}^{2}=k_{x}^{2}+k_{y}^{2}, \\
k_{ \pm}=k_{x} \pm i k_{y}, \quad T=E_{g}+B(2 F+1)\left(k_{\|}^{2}+k_{z}^{2}\right), \\
U=-B \gamma_{1}\left(k_{\|}^{2}+k_{z}^{2}\right), \quad V=-B \bar{\gamma}\left(k_{\|}^{2}-2 k_{z}^{2}\right),
\end{gathered}
$$

where $m_{0}$ is the electron mass. For the simplicity of the physical picture, we have taken the axial approximation $\bar{\gamma}=\left(\gamma_{2}+\gamma_{3}\right) / 2$ which makes the band structure isotropic in the $k_{x}, k_{y}$ plane. $E_{g}, E_{p}, F, \gamma_{1}, \gamma_{2}$, and $\gamma_{3}$ are materialspecific parameters. The basis functions are denoted as $\left(\left|\psi_{1}\right\rangle,\left|\psi_{2}\right\rangle\right) \Gamma_{6},\left(\left|\psi_{3}\right\rangle,\left|\psi_{4}\right\rangle,\left|\psi_{5}\right\rangle,\left|\psi_{6}\right\rangle\right) \Gamma_{8}$. Here we take the parameters of $\mathrm{HgTe}$ just for an illustration of the physics (Table I). The same physics should also happen in the recently discovered Heusler and Li-based ternary compounds which share similar band topologies. ${ }^{16-22}$

\section{TWO TYPES OF SURFACE STATES IN 3D SEMIMETALS}

Without strain, $\mathrm{HgTe}$ is a semimetal with an inverted band structure. At the $\Gamma$ point, the conduction band $\left[\Gamma_{8}\right.$ light hole $(\mathrm{LH})]$ touches the valence band $\left[\Gamma_{8}\right.$ heavy hole $\left.(\mathrm{HH})\right]$ and the $\Gamma_{6}$ is below the $\Gamma_{8}$ band (see right part of Fig. 1). In the Hamiltonian $H_{0}$ the band topology is solely determined by the parameter $E_{g}$. At the $\Gamma$ point, $\mathrm{LH}$ and $\mathrm{HH}$ are degenerate at energy $E=0$, and $\Gamma_{6}$ is at position $E=E_{g}$. So when $E_{g}>0$, the band structure is normal and insulating with a positive energy gap $\Delta=E_{g}$. When $E_{g}<0$, the band structure is inverted $\Gamma_{6}$ appears below $\mathrm{LH}$ and $\mathrm{HH}$. In this case, the system is semimetal since the bulk band gap $\Delta$ is always zero because of the $\mathrm{LH} / \mathrm{HH}$ degeneracy. We will show this semimetal is topologically nontrivial by showing that gapless surface states exist in the direct gap of $\mathrm{LH}$ and $\mathrm{HH}$. And by lifting the degeneracy of $\mathrm{LH}$ and $\mathrm{HH}$ by strain or finite confinement which makes $\Delta$ nonzero, the system instantly becomes a topological insulator. Whereas in trivial semimetals,

TABLE I. Band structure parameters of $\mathrm{HgTe}$ at $T=0 \mathrm{~K}^{25}$

\begin{tabular}{lccccc}
\hline \hline$E_{g}$ & $E_{P}=2 m_{0} P^{2} / \hbar^{2}$ & $F$ & $\gamma_{1}$ & $\gamma_{2}$ & $\gamma_{3}$ \\
\hline$-0.3 \mathrm{eV}$ & $18.8 \mathrm{eV}$ & 0 & 4.1 & 0.5 & 1.3 \\
\hline \hline
\end{tabular}

lifting the zero band gap only turns the systems into trivial band insulators.

To study the topological properties of this Hamiltonian we transform it into a tight-binding model on a cubic lattice, where such approximation substitutions are used:

$$
k_{i} \rightarrow \frac{1}{a} \sin \left(k_{i} a\right), \quad k_{i}^{2} \rightarrow \frac{2}{a^{2}}\left[1-\cos \left(k_{i} a\right)\right],
$$

here $k_{i}$ refers to $k_{x}, k_{y}$, and $k_{z}, a$ is the lattice constant which is taken to be $4 \AA$; in this work. This approximation is valid in the vicinity of the $\Gamma$ point.

Surface states reside only on the system surface, which will project larger LDOS on the surface than the bulk states. Thus the surface LDOS can be studied to identify the existence of surface states. The surface LDOS is given by $\rho(k)=$ $-\frac{1}{\pi} \operatorname{Tr} I m G_{00}(k)$, where $G_{00}$ is the retarded Green's function for the top layer of a 3D lattice. In a semi-infinite 3D system the surface Green's function can be obtained by means of the transfer matrix,

$$
\begin{gathered}
G_{00}=\left(E-H_{00}-H_{01} \mathbf{T}\right)^{-1}, \\
\mathbf{T}=\left(E-H_{00}-H_{01} \mathbf{T}\right)^{-1} H_{01}^{\dagger},
\end{gathered}
$$

where $H_{00}$ and $H_{01}$ are the matrix elements of the Hamiltonian within and between the layers (or supercells) and $\mathbf{T}$ is the transfer matrix. Usually Eq. (4) can be calculated iteratively

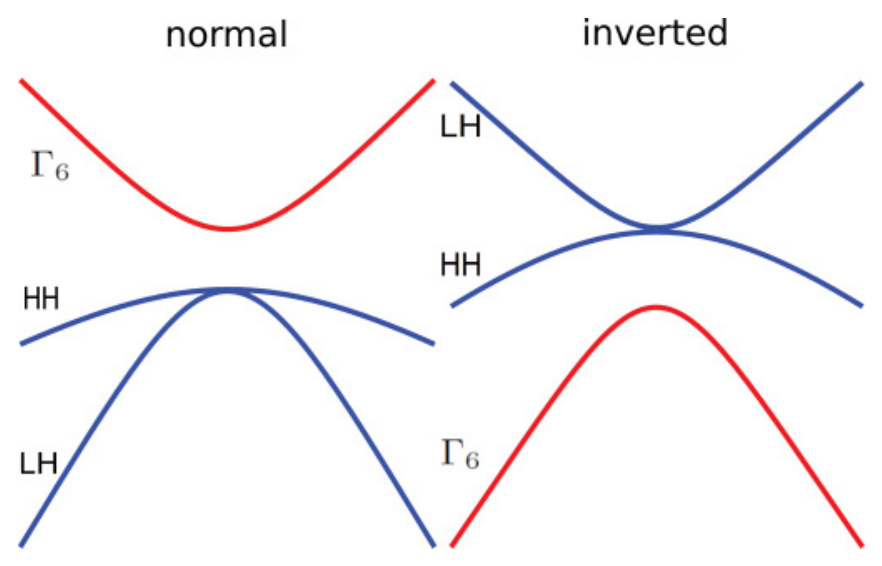

FIG. 1. (Color online) A schematic illustration of the band inversion between $\Gamma_{6}$ and $\Gamma_{8}$ the left is the normal case where the blue curve represents the $\mathrm{LH}$ and $\mathrm{HH}$ of the $\Gamma_{8}$ valence band, the right is the inverted case where the LH flips up and becomes the conduction band, the $\Gamma_{6}$ appears below the $\mathrm{HH}$ band. 

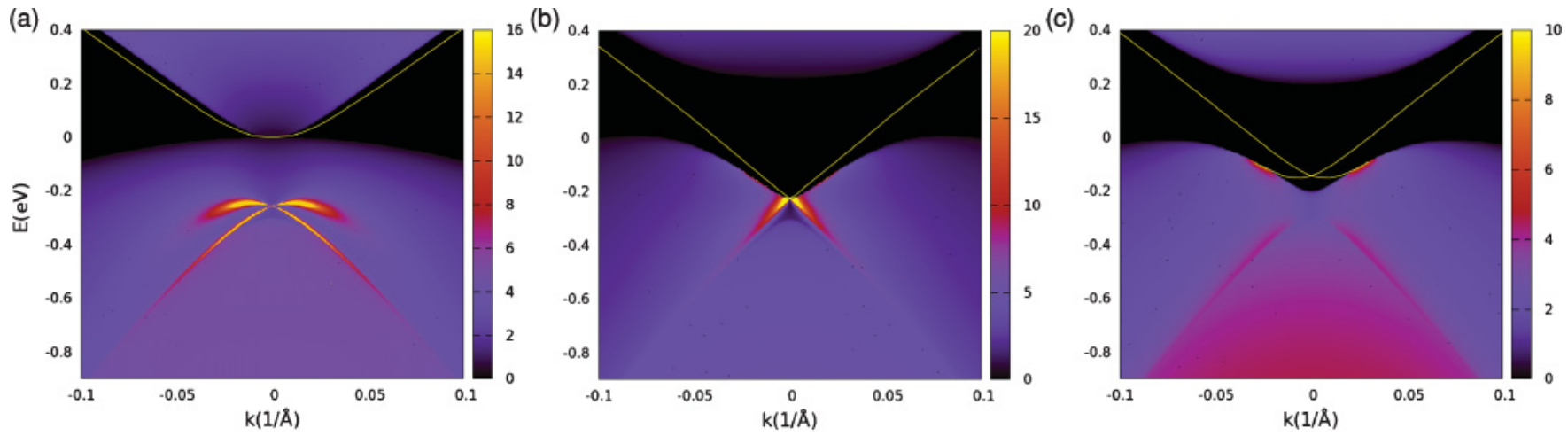

FIG. 2. (Color online) (a) Surface LDOS of 3D HgTe without strain, bright line in the direct gap between LH and HH $\Gamma_{8}$ bands indicates the first-type surface state, bright regions in the valence band indicates the second-type surface state, $E_{\Gamma_{6}}<E_{0}<E_{H H}=E_{L H}$. (b) An insulating band gap is opened with strain $T_{\epsilon}=0, U_{\epsilon}=0, V_{\epsilon}=-0.224 \mathrm{eV}$, the first- and second-type surface states become connected, $E_{\Gamma_{6}}<E_{H H}=E_{0}<E_{L H}$. (c) Same as (b) at $T_{\epsilon}=0.1 \mathrm{eV}, U_{\epsilon}=-0.05 \mathrm{eV}, V_{\epsilon}=-0.25 \mathrm{eV}$, note that the $\Gamma_{6}$ band has been inverted with the $\mathrm{HH} \Gamma_{8}$ band and thus appears in the middle of $\Gamma_{8}$ bands (LH and $\left.\mathrm{HH}\right)$, i.e., $E_{H H}<E_{\Gamma_{6}}<E_{0}<E_{L H}$. A highest LDOS limit is set and higher data points have been filtered to this limit for a clearer view of the whole spectrum.

until T converges, which is quite time consuming. Here we use a fast converging algorithm proposed by Sancho et al. to calculate the transfer matrix. ${ }^{26}$ In Fig. 2(a) we present the LDOS on an infinite $x y$ surface, where the $z$ dimension is semiinfinite. As expected, the LDOS clearly shows the existence of surface states between the LH and HH bands. Through further checking we find that these states indeed reside only on the surface boundary [see Fig. 3(a)]. Its spatial distribution is in the decaying form beneath the surface. Interestingly, we also find another kind of surface state submerging in the valence bands [bright crossing regions in the valence band of Fig. 2(a)]. It shows up between the inverted $\Gamma_{6}$ and $\mathrm{HH}$ $\Gamma_{8}$ bands. We confirm its surface-state nature by checking its spatial distribution along the $z$ direction [Figs. 3(b) and 3(c)]. It is found that the spatial distribution of this surface state has a very distinct form from that of the $\mathrm{LH}$ and $\mathrm{HH}$ bands. It bears the oscillating feature of LDOS of bulk states [see Fig. 3(d)] but is also clearly decaying beneath the surface. We call the former surface state as the first type and the latter the second type. Both types of surface-state project much larger LDOS on the surface than the bulk states. However, the first-type surface state is clearly decoupled from the bulk states while the second type is coupled with the valence bulk conduction states.

The spatial distribution can be obtained by enlarging the supercell when calculating the surface Green's function $G_{00}$. For example, take the first 50 layers as a unit cell, we can obtain the LDOS distribution in the first 50 layers. Figure 3(a) shows the spatial distribution of the first-type surface state at variant $k$. It shows that the closer to the $\Gamma$ point the wider the wavefunction distributes in space. Only away from the $\Gamma$ point, the first-type surface state shows strong localization on the surface. Close to the $\Gamma$ point, the LDOS distribution is bulk-like and can barely be recognized as surface state. In Fig. 2 the bright line indicating the first-type surface has been highlighted for a clearer view. The Fermi surface of 3D HgTe is close to the point where the conduction band touches the valence band. ${ }^{18,29}$ The first-type surface states at the Fermi surface distribute widely in the space. They are expected to make no significant contribution to the transport properties either. Without appropriate doping or gating it is difficult to detect the first-type surface states in 3D HgTe experimentally

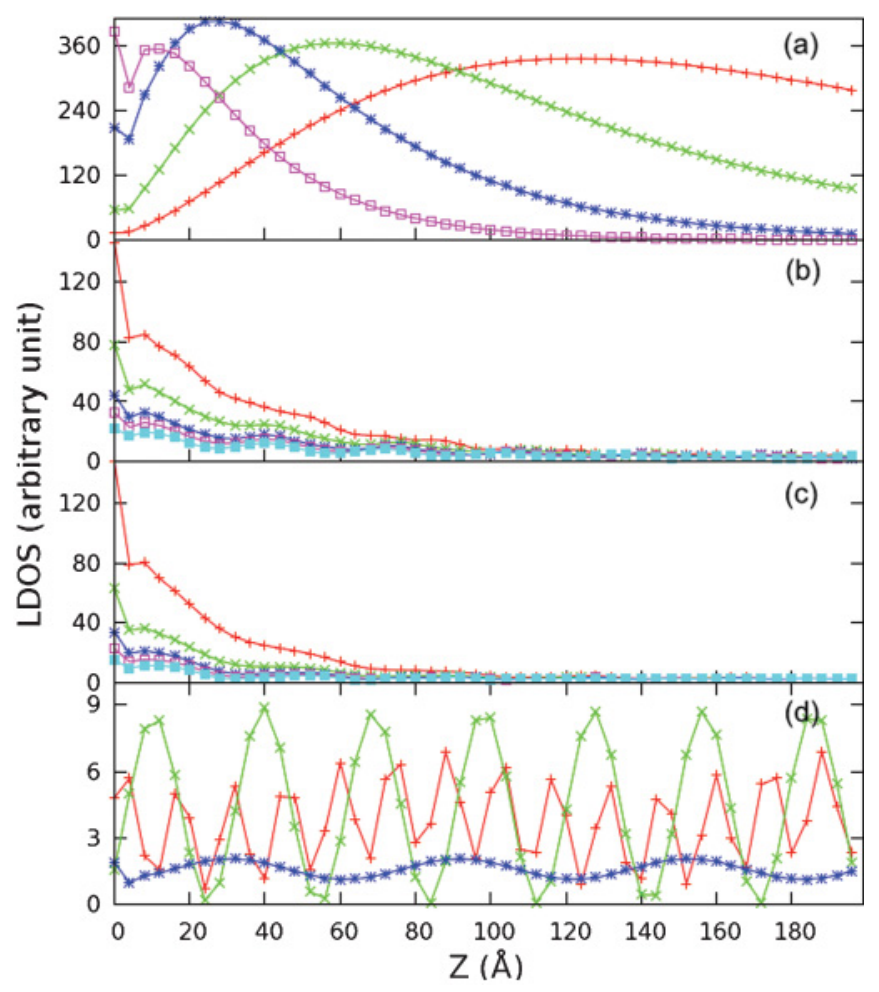

FIG. 3. (Color online) Surface state (ss) distribution along the $z$ direction. (a) First-type ss at $k=0.005$ (red, plus sign dot), 0.01 (green, cross dot), 0.02 (blue, star dot), 0.035 (pink, empty square dot) $1 / \AA$. (b) Second-type ss below the crossing point at $k=0.0066$ (red, plus sign dot), 0.01 (green, cross dot), 0.014 (blue, star dot), 0.017 (pink, empty square dot), 0.022 (cyan, filled square dot) $1 / \AA$. (c) Second-type ss above the crossing point at $k$, same as in (b). (d) Bulk states at $k=0.00661 / \AA$ and $E=-0.4 \mathrm{eV}$ (red, plus sign dot), $-0.1 \mathrm{eV}$ (green, cross dot), $0.2 \mathrm{eV}$ (blue, star dot). 
either through angle-resolved photoemission spectroscopy (ARPES) or transport measurements.

Unlike the first type surface states which show up as welldefined sharp lines in the energy spectrum, the second-type surface state appears above the boundary of the $\Gamma_{6}$ band as two crossing bright regions submerging in the valence bands in the energy spectrum. Its LDOS on the surface is the highest close to the $\Gamma$ point. Away from the $\Gamma$ point, the surface LDOS becomes smaller and finally those states merge into the bulk states. Also unlike the first-type surface state whose distribution width becomes very large when approaching the $\Gamma$ point, the second-type surface state's distribution width in space does not significantly depend on the momentum. At the $\Gamma$ point, the second-type surface-state energy and wave function is exactly solvable from the Hamiltonian. We will show that this point is the crossing point of the surface state Dirac cone (see text below). Beneath the surface, the LDOS quickly decays in an oscillatory way. For comparison, we also plot out the bulk states LDOS which is oscillatory and extended all over the space without decaying [Fig. 3(d)]. Since from the LDOS the second-type surface state can be easily distinguished from the bulk states, we believe they may be easily detected from the ARPES measurements as well.

\section{ORIGIN OF SURFACE STATES AND STRAIN-INDUCED BAND GAP}

The origin of the two types of surface states can be understood intuitively in the following way. If we divide the Hamiltonian into two subspaces which are spanned by two groups of basis: $\mathcal{P}_{1}=\left\{\psi_{1}, \psi_{4}, \psi_{2}, \psi_{5}\right\}$ and $\mathcal{P}_{2}=\left\{\psi_{3}, \psi_{6}\right\}$, it is amazing that the effective Hamiltonian in the subspace $\mathcal{P}_{1}$ is quite similar to the effective model proposed for a $3 \mathrm{D}$ topological insulator. ${ }^{14}$ The Hamiltonian after arranging the basis as $\left[\mathcal{P}_{1}\left(\psi_{1}, \psi_{4}, \psi_{2}, \psi_{5}\right), \mathcal{P}_{2}\left(\psi_{3}, \psi_{6}\right)\right]$ reads

$$
H_{0}^{\prime}=\left(\begin{array}{lcc}
T & \sqrt{\frac{2}{3}} P k_{z} & 0 \\
\sqrt{\frac{2}{3}} P k_{z} & U-V & -\frac{1}{\sqrt{6}} P k_{-} \\
0 & -\frac{1}{\sqrt{6}} P k_{+} & T \\
\frac{1}{\sqrt{6}} P k_{+} & 0 & \sqrt{\frac{2}{3}} P k_{z} \\
-\frac{1}{\sqrt{2}} P k_{-} & 2 \sqrt{3} \bar{\gamma} B k_{-} k_{z} & 0 \\
0 & \sqrt{3} \bar{\gamma} B k_{+}^{2} & \frac{1}{\sqrt{2}} P k_{+}
\end{array}\right.
$$

Without considering the coupling between $\mathcal{P}_{1}$ and $\mathcal{P}_{2}$ subspaces, $\mathcal{P}_{1}$ gives the LH (conduction) and $\Gamma_{6}$ (valence) band, plus a gapless Dirac cone of surface states between their gap; $\mathcal{P}_{2}$ gives the $\mathrm{HH}$ (valence) which overlaps with the surface-state Dirac cone and $\Gamma_{6}$ band. After turning on the coupling between the two subspaces, the surface-state Dirac cone becomes separated into two parts. One part appears in the direct gap between $\mathrm{LH}$ and $\mathrm{HH}$, the other submerges into the $\mathrm{HH}$ of valence band. In this sense, our results are very similar to an earlier work which discusses interface states in $\mathrm{Hg}_{1-x} \mathrm{Cd}_{x} \mathrm{Te}$ heterojunctions. ${ }^{3}$

The $\Gamma_{8}$ degeneracy at the $\Gamma$ point makes 3D HgTe a semimetal. To lift the degeneracy and open an insulating gap at the Fermi energy, which makes the system a true 3D TI, we consider applying a uniaxial strain along the (001) axis. ${ }^{11,28}$ According to Ref. 23, the additional strain induced Hamiltonian is introduced as [in the original basis order as in Eq. (1)]

$$
H_{s}=\left(\begin{array}{cccccc}
T_{\epsilon} & 0 & 0 & 0 & 0 & 0 \\
0 & T_{\epsilon} & 0 & 0 & 0 & 0 \\
0 & 0 & U_{\epsilon}+V_{\epsilon} & 0 & 0 & 0 \\
0 & 0 & 0 & U_{\epsilon}-V_{\epsilon} & 0 & 0 \\
0 & 0 & 0 & 0 & U_{\epsilon}-V_{\epsilon} & 0 \\
0 & 0 & 0 & 0 & 0 & U_{\epsilon}+V_{\epsilon}
\end{array}\right),
$$

where the $T_{\epsilon}, U_{\epsilon}$, and $V_{\epsilon}$ are strain-induced interaction terms. To illustrate the physical picture more clearly, we have made these terms artificially large in this work. At the $\Gamma$ point, the boundary for the $\Gamma_{6}$ band is $E_{\Gamma_{6}}=E_{g}+T_{\epsilon}$, for the $\mathrm{HH}$ band the boundary is $E_{H H}=U_{\epsilon}+V_{\epsilon}$, and for the LH band the boundary is $E_{L H}=U_{\epsilon}-V_{\epsilon}$. In the strain-free condition, $E_{\Gamma_{6}}<E_{L H}=E_{H H}=0$. However, under strain, the relative position of the three is subject to change and is determined by the strain terms.

Surface state at the crossing point of the Dirac cone is easily solvable directly from the Hamiltonian $H_{0}+H_{s}$, where $k_{x}=k_{y}=0 .{ }^{30}$ Using the boundary condition $\psi(z=0)=0$ and $\psi(z=-\infty)=0$, we get the eigenenergy for the surface state:

$$
\begin{aligned}
E_{0} & =C+\frac{D_{1} M}{B_{1}} \\
& =\frac{\left(E_{g}+T_{\epsilon}\right)\left(\gamma_{1}+2 \bar{\gamma}\right)+\left(U_{\epsilon}-V_{\epsilon}\right)(2 F+1)}{\gamma_{1}+2 \bar{\gamma}+2 F+1},
\end{aligned}
$$

where

$$
\begin{gathered}
C=\frac{E_{g}+T_{\epsilon}+U_{\epsilon}-V_{\epsilon}}{2}, \quad M=\frac{E_{g}+T_{\epsilon}-U_{\epsilon}+V_{\epsilon}}{2}, \\
D_{1}=\frac{B}{2}\left(2 F+1-\gamma_{1}-2 \bar{\gamma}\right),
\end{gathered}
$$




$$
B_{1}=-\frac{B}{2}\left(2 F+1+\gamma_{1}+2 \bar{\gamma}\right) .
$$

And the wave function is

$$
\varphi=\frac{1}{\sqrt{2}}\left(\begin{array}{l}
i \sqrt{\frac{D_{+}}{B_{1}}} \\
\sqrt{-\frac{D_{-}}{B_{1}}}
\end{array}\right) c\left(e^{\lambda_{1} z}-e^{\lambda_{2} z}\right),
$$

with the basis $\psi_{1}$ and $\psi_{4}$ or $\psi_{2}$ and $\psi_{5}$, where

$$
\begin{gathered}
\lambda_{1,2}^{2}=\frac{D_{2} \pm \sqrt{D_{2}^{2}-D_{3}}}{2 D_{+} D_{-}}, \quad \text { with } \\
D_{2}=-\left[A_{1}^{2}+D_{+}\left(E_{0}-L_{1}\right)+D_{-}\left(E_{0}-L_{2}\right)\right], \\
D_{3}=4 D_{+} D_{-}\left(E_{0}-L_{1}\right)\left(E_{0}-L_{2}\right),
\end{gathered}
$$

$D_{ \pm}=D_{1} \pm B_{1}, \quad L_{1}=C+M, \quad L_{2}=C-M$, and $A_{1}=$

$\sqrt{\frac{2}{3}} P$. For the surface-state solution to exist, it is required that

$$
M B_{1}>0 .
$$

This condition is easily satisfied in an inverted band structure without strain, where $E_{g}<0$.

In Figs. 2(b) and 2(c), the spectrum after adding the strain interaction is plotted. An insulating gap is opened between the $\mathrm{LH}$ and $\mathrm{HH} \Gamma_{8}$ bands. Meanwhile, both the first- and second-type surface states go through an evolution. At a critical point [Fig. 2(b)] the crossing point can move to the top of the valence band at the $\Gamma$ point and both types of surface states become connected with each other. On further increasing the strain strength, the crossing point jumps out of the valence band and sits in the band gap. At this stage, we find only one kind of surface state appearing in the insulating gap [Fig. 2(c)]. The spectrum of surface state forms a gapless Dirac cone at the $\Gamma$ point and the system becomes a typical 3D TI. Notice that Fig. 2(c) closely resembles the band structure of $3 \mathrm{D} \mathrm{TI} \mathrm{Bi}_{2} \mathrm{Se}_{3}$ and $\mathrm{Bi}_{2} \mathrm{Te}_{3} .{ }^{14,15}$ Although Fig. 2 is obtained from the tight binding model on a lattice, we find the energy of the crossing point agrees with the exact solution quite precisely.

\section{EDGE STATES IN 2D THIN FILMS}

\section{A. Quasi-2D lattice model}

It is, however, technically difficult applying strong enough strains to make a semimetal insulating in experimental conditions. The finite-size effect can open a gap in the bulk states at the $\Gamma$ point but will also open a gap in the surface states. ${ }^{27,30}$ Instead of considering the surface states, we make the 3D topologically nontrivial semimetals into thin films and study their edge effects in the strain-free condition. The same lattice model is used as in the 3D case, but now the $z$ dimension is of finite thickness $L$. We study the LDOS on the side surface of a thin film where $x$ is infinite and $y$ is semi-infinite. In this case, only $k_{x}$ is a good quantum number. In Fig. 4, the LDOS on the film edge surface for various film thickness is plotted. In the thick film limit, the band gap opened by the finite-size effect is not obvious. The spectrum still resembles that of the 3D case [Figs. 4(a) and 4(b)]. Surface states still
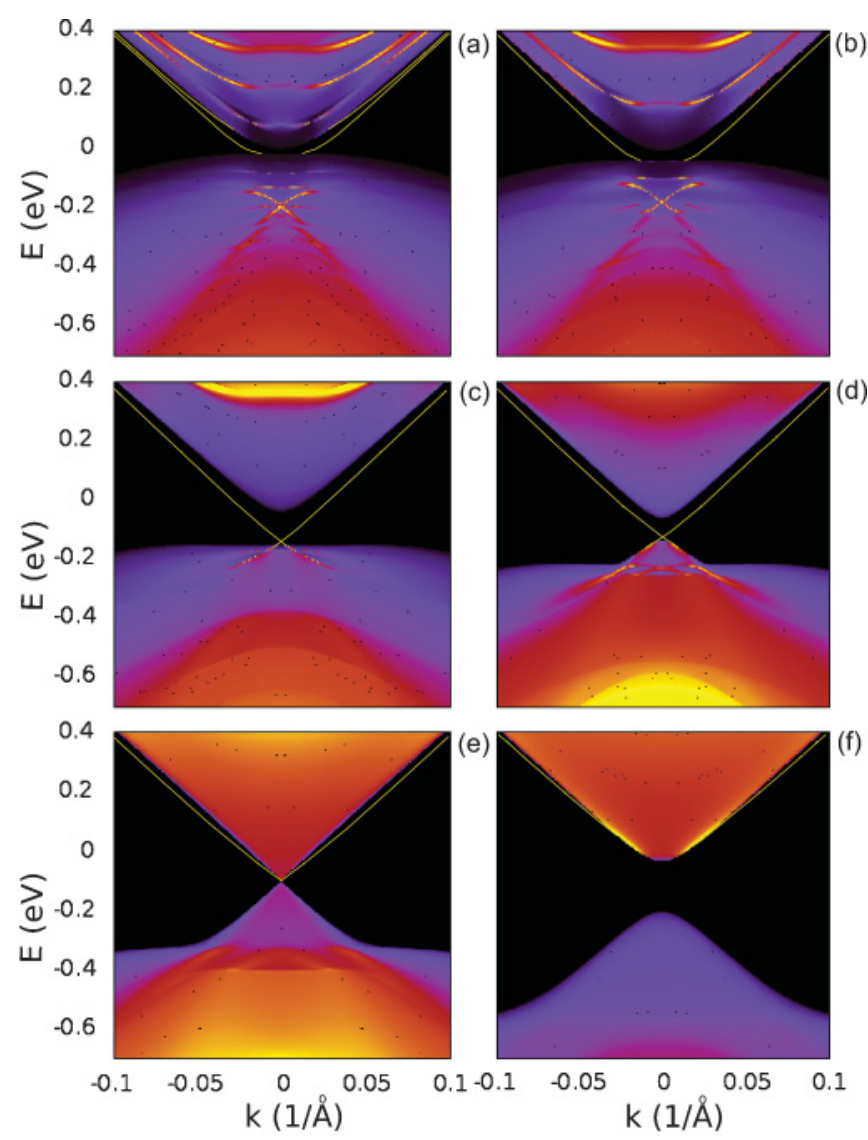

FIG. 4. (Color online) LDOS at the edge of thin films at different thickness calculated with the $3 \mathrm{D}$ lattice model. (a) $L=$ $116 \AA$; (b) $L=76 \AA$; (c) $L=36 \AA$; (d) $L=28 \AA$; (e) $L=20 \AA$; (f) $L=12 \AA$;.

show residuals on the spectrum. The finite confinement of $z$ causes energy-level discretization as in a quantum well, which induces a series of sub-bands appearing in the energy spectrum as layered structures. When the film is thinned down the discretized energy level spacing increases. A pair of edge states with linear dispersion are found in the band gap [Fig. 4(c) and 4(d)]. The system becomes a 2D topological insulator. When the film is thinner than $20 \AA$, another transition happens, the system becomes trivial. The edge states disappear from the band gap.

\section{B. Quantum well approximation and 2D lattice model}

When the film is thin enough, the finite-size-caused band gap becomes obvious. In this case the finite-confinementinduced sub-bands are far away from the low-energy regime. We can then use the quantum well approximation $\left\langle k_{z}\right\rangle=$ $0,\left\langle k_{z}^{2}\right\rangle \simeq(\pi / L)^{2} \cdot{ }^{31}$ Using these relations in the Hamiltonian in Eq. (1), and choosing the basis set in the sequence $\left(\left|\psi_{1}\right\rangle\right.$, $\left.\left|\psi_{3}\right\rangle,\left|\psi_{5}\right\rangle,\left|\psi_{2}\right\rangle,\left|\psi_{6}\right\rangle,\left|\psi_{4}\right\rangle\right)$, we can obtain a two-dimensional six-band Kane model

$$
H(\mathbf{k})=\left(\begin{array}{cc}
h(\mathbf{k}) & 0 \\
0 & h^{*}(-\mathbf{k})
\end{array}\right)
$$


where

$$
h(\mathbf{k})=\left(\begin{array}{ccc}
E_{g}+B(2 F+1)\left(k_{\|}^{2}+\left\langle k_{z}^{2}\right\rangle\right) & -\frac{1}{\sqrt{2}} P k_{+} & \frac{1}{\sqrt{6}} P k_{-} \\
-\frac{1}{\sqrt{2}} P k_{-} & -\left(\gamma_{1}+\bar{\gamma}\right) B k_{\|}^{2}-\left(\gamma_{1}-2 \bar{\gamma}\right) B\left\langle k_{z}^{2}\right\rangle & \sqrt{3} \bar{\gamma} B k_{-}^{2} \\
\frac{1}{\sqrt{6}} P k_{+} & \sqrt{3} \bar{\gamma} B k_{+}^{2} & -\left(\gamma_{1}-\bar{\gamma}\right) B k_{\|}^{2}-\left(\gamma_{1}+2 \bar{\gamma}\right) B\left\langle k_{z}^{2}\right\rangle
\end{array}\right) .
$$

The system keeps time-reversal symmetry, and the representation of the symmetry operation in the new set of bases is given by $T=\mathcal{K} \cdot i \sigma^{y} \otimes I_{3 \times 3}$, where $\mathcal{K}$ is the complex conjugation operator, $\sigma^{y}$ and $I$ denote the Pauli matrix and unitary matrix in the spin and orbital space, respectively. We can study the two blocks separately since they are time-reversal counterparts of each other. Here we focus on the upper block first. At $k_{x}=0$, the boundaries of $\Gamma_{6}, \mathrm{LH}$, and $\mathrm{HH}$ are at $E=E_{g}+B(2 F+1)\left\langle k_{z}^{2}\right\rangle, E=-\left(\gamma_{1}-2 \bar{\gamma}\right) B\left\langle k_{z}^{2}\right\rangle$, and $E=-\left(\gamma_{1}+2 \bar{\gamma}\right) B\left\langle k_{z}^{2}\right\rangle$, which are controllable by choosing film thickness $L$. When $L$ decreases from the thick limit down to $L \approx 30 \AA$ the $\Gamma_{6}$ band flips up and exchanges position with $\mathrm{HH}$, the system is still nontrivial. Further down to $L \approx 20 \AA$, $\Gamma_{6}$ flips up and exchanges with the conduction band (LH) [see Fig. 5(e)]. The band structure then becomes trivial just as the illustrated case in Fig. 1. This rough picture serves as an intuitive understanding of the topological transition and edge-state formation in the thin films. It also agrees with the result we obtained with the 3D lattice model in Fig. 4. In the thick film limit, the bulk band gap is always between the LH
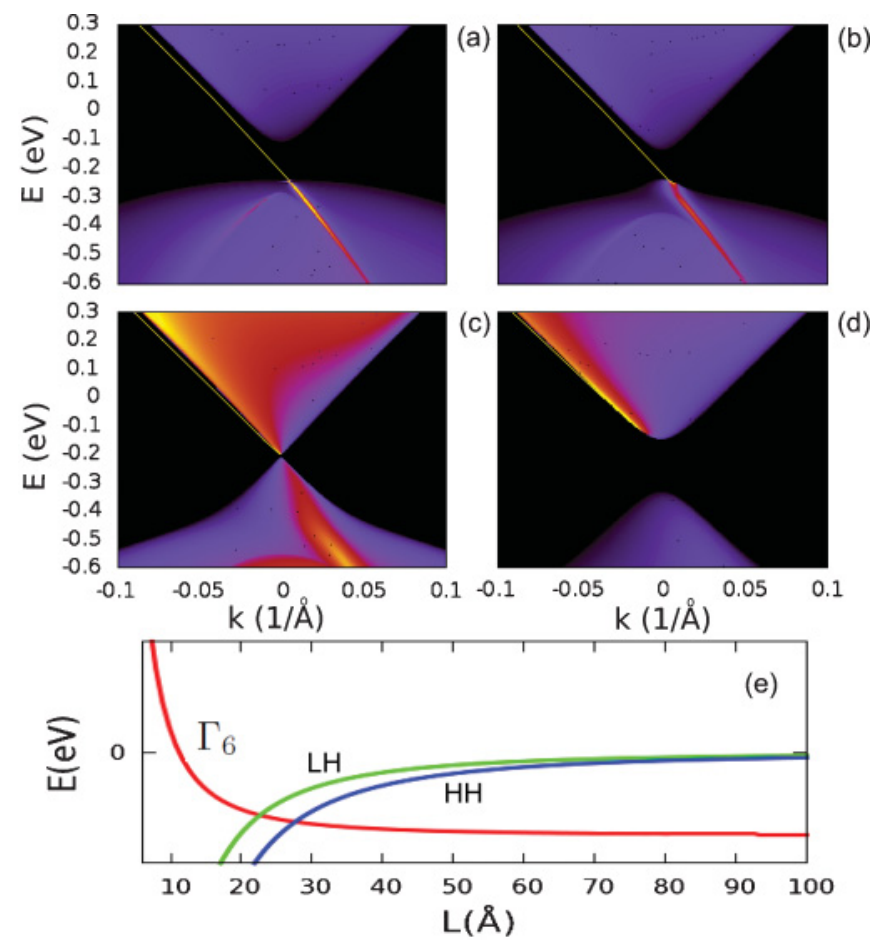

FIG. 5. (Color online) LDOS at the edge of thin films at different thickness using the Hamiltonian (9) with 2D lattice model. (a) $L=28 \AA$; (b) $L=25 \AA$; (c) $L=20 \AA$; (d) $L=16 \AA$; (e) illustration of the position change of $\mathrm{LH}, \mathrm{HH}$, and $\Gamma_{6}$ bands as film thickness is varied. and $\mathrm{HH}$ derived states while in thin limit it is between the $\Gamma_{6}$ and LH derived states. In the thick-film limit, surface states (on top and bottom surfaces) and edge states (on side surfaces) coexist. The first-type surface states on the top/bottom surfaces become the effective bulk states of the film appearing as a conduction band minimum. The edge states in the thinner films actually evolve out from the surface states on the side surfaces, whose nature is 2D instead of 1D.

Using the same approximation as in Eq. (2), we can transform $h(\mathbf{k})$ into a tight-binding model on a 2D lattice. In Fig. 5 we show the LDOS on the edge of a semi-infinite film for $h(\mathbf{k})$. The finite-size gap agrees with that obtained with the 3D lattice in Fig. 4 well. When $L>20 \AA$, the edge states are found connecting the valence and conduction bands. After the system becomes trivial when $L<20 \AA$, the edge states do not cross the band gap anymore, instead they only attach to the conduction band. At the critical point $L=20 \AA$, the valence band and conduction band touch and form a linear Dirac cone at the low-energy regime, which is shown in both Figs. 4 and 5. This shows that by controlling the film thickness, it is possible to obtain a single-valley Dirac cone for each spin block without using the topological surface states. ${ }^{32}$ Notice that in Fig. 5 we can also see the edge states submerging in the valence bands, which is similar to the case of second-type surface states discussed previously. We call them the second-type edge states.

\section{Chern number description of thin-film band topology}

In the quantum-Hall effect (QHE), bulk-edge correspondence tells us that a nonzero Thouless-Kohmoto-Nightingaleden Nijs (TKNN) integer, summed by the Chern number of occupied bands, is closely related to the presence of edge states on the sample boundaries. ${ }^{33,34}$ For the time-reversal invariant systems which belong to the universality class of zero-charge Chern number, the $Z_{2}$ index is then introduced to characterize the topologically nontrivial states. ${ }^{7,8}$ However, when the Hamiltonian of the system is composed of timereversal diagonal blocks, ${ }^{9}$ the $Z_{2}$ invariant can be identified with the parity of the Chern number for each block. ${ }^{11}$ Focusing on the upper block of the thin-film Hamiltonian Eq. (8) [i.e. $h(\mathbf{k})]$, we can discuss the topology of the band structure. To identify the existence of edge states, we can calculate the first Chern number of each band and sum up for all occupied bands. The Berry curvature for each band is defined as ${ }^{35,36}$

$$
\Omega_{n}(k)=i\left(\left\langle\frac{\partial u_{n, k}}{\partial k_{x}} \mid \frac{\partial u_{n, k}}{\partial k_{y}}\right\rangle-\left\langle\frac{\partial u_{n, k}}{\partial k_{y}} \mid \frac{\partial u_{n, k}}{\partial k_{x}}\right\rangle\right) .
$$

Then the Chern number is computed by integrating the Berry curvature over the first Brillouin Zone (BZ): $C_{n}=$ $\frac{1}{2 \pi} \int_{B Z} d^{2} k \Omega_{n}(k)$. For each block of the 2D lattice model, the 
Chern number can be given to each of the three bands. Tuning the thickness of the quantum well $L$ can inverse the band structure near the $\Gamma$ point, resulting in the change of Chern number for the touching bands. Several critical thicknesses for $L$ can be found when there is a band touching at the $\Gamma$ point: $L \approx 30 \AA$ and $L \approx 20 \AA$. The numerical result of this integral divides the parameter space into three regions: for $L \in(30 \AA,+\infty)$, Chern number $C=1,0,-1$ arranged in sequence from the lowest energy band to the highest (the same for the latter two cases); for $L \in(20 \AA, 30 \AA)$, Chern number $C=2,-1,-1$, and for $L \in(0,20 \AA)$, Chern number $C=2,-2,0$. When the Fermi level lies between the highest and the middle energy band, the TKNN integer $N=1$ for $L \in(20 \AA,+\infty)$ and $N=0$ for $L \in(0,20 \AA)$. The former case is topologically nontrivial [referred to in Figs. 5(a) and 5(b)] and the latter is trivial [referred to in Fig. 5(d)].

\section{QSHE AND DISORDER-ENHANCED SPIN-HALL EFFECT}

The edge state picture in thin films can be verified with explicit Landauer-Büttiker calculations. ${ }^{37,38}$ We construct a four-terminal Hall bar device using the tight binding version of the Hamiltonian (8). Because the system keeps time reversal symmetry (TRS), we need only consider a single block of the $6 \times 6$ Hamiltonian. The transverse charge conductance of each sub-block is defined as $G_{t c}=G_{12}-G_{14} . G_{i j}$ denotes the conductance between terminal $i$ and $j$, which is given by $G_{i j}=\frac{e^{2}}{h} \operatorname{Tr}\left[\Gamma_{i} G^{R} \Gamma_{j} G^{A}\right] . \Gamma_{i}=i\left[\sum_{i}^{R}-\sum_{i}^{A}\right]$ is the spectrum function of lead $i$. $G^{R / A}$ is the retarded and advanced Green's function of the sample which has taken into account the four semi-infinite leads through the self-energy $\sum_{i}^{R / A}: G^{R / A}=$ $\left[E-H_{c}-\sum_{i=1}^{4} \sum_{i}^{R / A}\right]^{-1} . H_{c}$ denotes the Hamiltonian of the shaded region of the sample. ${ }^{38-40}$ In a real system with TRS, the transverse charge conductance calculated here corresponds to the transverse "spin" conductance, which is defined as $G_{t s}=$ $G_{t c}^{\uparrow}-G_{t c}^{\downarrow}$. The longitudinal conductance is defined as $G_{l}=$ $G_{13}^{\uparrow}+G_{13}^{\downarrow}$. However, it should be noted that the "spin" up or down here is only an index of each Hamiltonian sub-block and does not mean real spin. The bases of each sub-block are hybrids of $j= \pm 1 / 2$ and $j= \pm 3 / 2$ states of $\Gamma_{6}$ and $\Gamma_{8}$ bands. ${ }^{9}$

In Fig. 6, we show the result for a film whose thickness is $25 \AA$. In the clean limit, i.e., without disorder, in the finite-size induced band gap, the $G_{l}$ vanishes, $G_{t s}$ is quantized as $G_{t s}=2 e^{2} / h$ (with $G_{12}^{\uparrow}=G_{14}^{\downarrow}=e^{2} / h, G_{14}^{\uparrow}=G_{12}^{\downarrow}=0$ ), which proves the existence of helical edge states in the thin films and indicates the existence of quantum spin-Hall effect (QSHE). ${ }^{41-45}$ In the conduction band, the edge states coexist with the bulk states. $G_{t s}$ there is not quantized, which implies that the bulk states themselves carry transverse spin conductance that partly cancels out the quantized spin-Hall conductance carried by the edge state channels. In the valence band, similarly, the second-type edge states coexist with bulk states. Notice that the confinement in the $x, y$ dimension also generates a finite-size gap in the edge state itself, which is indicated by the arrow in Fig. 6 where $G_{t s}$ vanishes.

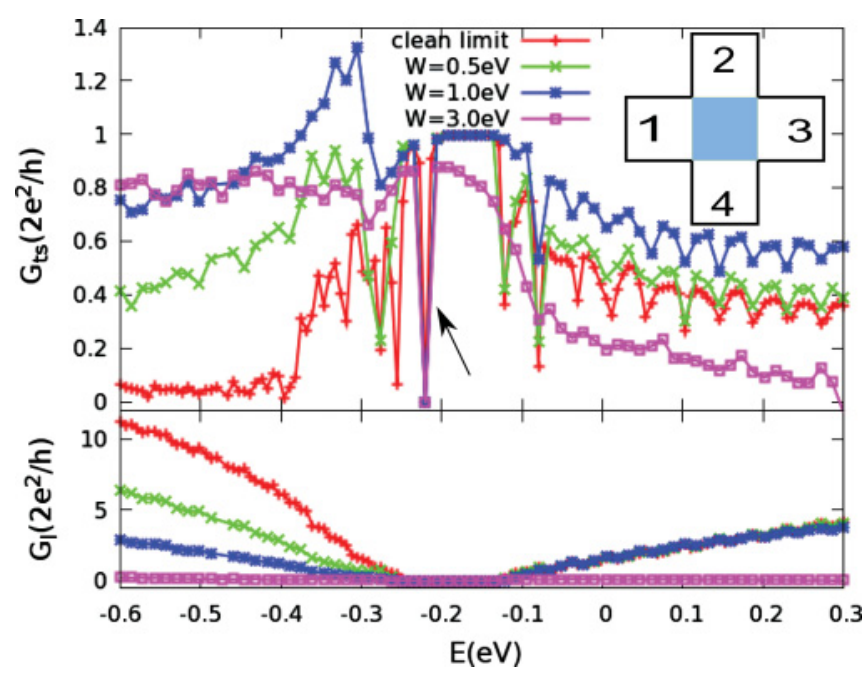

FIG. 6. (Color online) Four terminal Landauer-Büttiker calculations for thin films. Four identical semi-infinite leads are attached to the cross-bar device, which is shown schematically in the inset. Disorders are put on the central area (shaded areas). 200 disorder samples are taken for each disorder strength $W$. The arrow indicates the position of finite-size gap of the helical edge states.

Disorder is recently known to play an important role in some exotic phenomena in TIs. ${ }^{46,47}$ In real materials, dirty impurities are inevitable. It is even possible to control them artificially. ${ }^{48,49}$ Here we consider the effect of disorder impurities to the transport properties of the thin films. Andersontype white noise is introduced on the lattice model, which are spin independent on-site random potentials in the range [ $-W / 2, W / 2]$. TRS is not violated by disorder. In Fig. 6 we show the transverse spin conductance $G_{t s}$ of the four-terminal device at various disorder strength $W$. The quantized $G_{t s}$ in the band gap shows robustness against modest disorder strength, which is expected because the disorder we apply does not couple the TRS sub-blocks of the Hamiltonian and thus brings no backscattering between different spin-edge channels. ${ }^{7,9}$ Interestingly, $G_{t s}$ is significantly enhanced in both conduction and valence bands even with weak disorder. While $G_{t s}$ quickly becomes suppressed in the conduction band when disorder increases, it still remains enhanced in the valence band. Notice that with modest disorder strength $(3 \mathrm{eV}$ in Fig. 6), the longitudinal conductance $G_{l}$ already vanishes in the low-energy regime, but the transverse-spin conductance remains enhanced in the valence band. Therefore with modest disorder strength, we expect a strong-disorder-enhanced spinHall effect in the valence band, which would manifest itself through strong signals in nonlocal measurements. In a very recent experiment a similar observation of the SHE difference in valence and conduction bands has been reported with $\mathrm{HgTe}$ quantum wells. ${ }^{50}$

\section{SUMMARY}

We have demonstrated that the six-band Kane model can be utilized to study the topological properties of both 3D and thin-film realistic materials. By choosing proper model parameters, it describes the band structure well in the lowenergy regime and captures the essential band topological 
features sufficiently. By calculating the LDOS on the system boundary, the existence of topological surface states is explicitly demonstrated. Using the model parameters of $\mathrm{HgTe}$, we show that even though the system in semimetal surface states already exists in the material as long as the band structure is inverted. We also find in the strain-free condition, surface states are divided into two parts in the spectrum, each of different characteristics. We demonstrate that uniaxial strains can generate an insulating gap and transform the semimetals into true TIs, in which the gapless Dirac cone of surface states is found in the bulk band gap. Because of the similar band structures and band topologies, we expect that the same physics applies to the recently discovered Heusler-related and Li-based intermetallic ternary compounds, which are mainly topologically nontrivial semimetals or metals.
We also demonstrate a crossover from a 3D topological semimetal to 2D QSHE insulators. In the thin films made of these materials, we predict the existence of helical edge states and QSHE in the strain-free condition. And finally we show that disorder plays an important role in the transport properties in the thin films. It significantly enhances the SHE in the valence bands.

\section{ACKNOWLEDGMENTS}

This work was supported by the Research Grant Council of Hong Kong under Grant Nos. HKU 7037/08P, HKUST3/CRF/09 and in part by a Hong Kong UGC Special Equipment Grant (SEG HKU09).
${ }^{1}$ J. E. Moore, Nature (London) 464, 194 (2010).

${ }^{2}$ B. A. Volkov and O. A. Pankratov, JETP Lett. 42, 178 (1985).

${ }^{3}$ O. A. Pankratov, S. V. Pakhomov, and B. A. Volkov, Solid State Commun. 61, 93 (1987).

${ }^{4}$ E. Fradkin, E. Dagotto, and D. Boyanovsky, Phys. Rev. Lett. 57, 2967 (1986).

${ }^{5}$ V. Korenman and H. D. Drew, Phys. Rev. B 35, 6446 (1987).

${ }^{6}$ D. Agassi and V. Korenman, Phys. Rev. B 37, 10095 (1988).

${ }^{7}$ C. L. Kane and E. J. Mele, Phys. Rev. Lett. 95, 146802 (2005).

${ }^{8}$ X. L. Qi, T. L. Hughes, and S.-C. Zhang, Phys. Rev. B 78, 195424 (2008).

${ }^{9}$ B. A. Bernevig, T. L. Hughes, and S. C. Zhang, Science 314, 1757 (2006).

${ }^{10}$ L. Fu, C. L. Kane, and E. J. Mele, Phys. Rev. Lett. 98, 106803 (2007).

${ }^{11}$ L. Fu and C. L. Kane, Phys. Rev. B 76, 045302 (2007).

${ }^{12}$ M. Konig, S. Wiedmann, C. Brune, A. Roth, H. Buhmann, L. W. Molenkamp, X. L. Qi, and S. C. Zhang, Science 318, 766 (2007).

${ }^{13}$ D. Hsieh, D. Qian, L. Wray, Y. Xia, Y. S. Hor, R. J. Cava, and M. Z. Hasan, Nature (London) 452, 970 (2008).

${ }^{14}$ H. J. Zhang, C. X. Liu, X. L. Qi, X. Dai, Z. Fang, and S. C. Zhang, Nature Phys. 5, 438 (2009).

${ }^{15}$ Y. Xia, D. Qian, D. Hsieh, L. Wray, A. Pal, H. Lin, A. Bansil, D. Grauer, Y. S. Hor, R. J. Cava, and M. Z. Hasan, Nature Phys. 5, 398 (2009).

${ }^{16}$ D. Xiao, Y. Yao, W. Feng, J. Wen, W. Zhu, X. Q. Chen, G. M. Stocks, and Z. Zhang, Phys. Rev. Lett. 105, 096404 (2010).

${ }^{17}$ M. Franz, Nat. Mater. 9, 536 (2010).

${ }^{18}$ S. Chadov, X. L. Qi, J. Kubler, G. H. Fecher, C. Felser, and S. C. Zhang, Nat. Mater. 9, 541 (2010).

${ }^{19}$ H. Lin, L. A. Wray, Y. Xia, S. Jia, R. J. Cava, A. Bansil, and M. Z. Hasan, Nat. Mater. 9, 546 (2010).

${ }^{20}$ H. Lin, R. S. Markiewicz, L. A. Wray, L. Fu, M. Z. Hasan, and A. Bansil, Phys. Rev. Lett. 105, 036404 (2010).

${ }^{21}$ H. Lin, L. A. Wray, Y. Xia, S. Jia, R. J. Cava, A. Bansil, and M. Z. Hasan, e-print arXiv:1004.0999 (2010).

${ }^{22}$ B. Yan, C. X. Liu, H. J. Zhang, C. Y. Yam, X. L. Qi, T. Frauenheim, and S. C. Zhang, e-print arXiv:1003.0074 (2010).
${ }^{23} \mathrm{R}$. Winkler, Spin-Orbit Coupling Effects in Two-Dimensional Electron and Hole Systems (Springer, Berlin, 2003).

${ }^{24}$ E. O. Kane, J. Phys. Chem. Solids 1, 249 (1957).

${ }^{25}$ E. G. Novik, A. Pfeuffer-Jeschke, T. Jungwirth, V. Latussek, C. R. Becker, G. Landwehr, H. Buhmann, and L. W. Molenkamp, Phys. Rev. B 72, 035321 (2005).

${ }^{26}$ M. P. L. Sancho, J. M. L. Sancho, and J. Rubio, J. Phys. F: Met. Phys. 14, 1205 (1984).

${ }^{27}$ B. Zhou, H. Z. Lu, R. L. Chu, S. Q. Shen, and Q. Niu, Phys. Rev. Lett. 101, 246807 (2008).

${ }^{28}$ X. Dai, T. L. Hughes, X. L. Qi, Z. Fang, and S. C. Zhang, Phys. Rev. B 77, 125319 (2008).

${ }^{29}$ I. A. Tsidikovski, Electron Spectrum of Gapless Semiconductors, Springer Series in Solid State Sciences, Vol. 116 (Springer-Verlag, Berlin, 1997).

${ }^{30}$ W. Y. Shan, H. Z. Lu, and S. Q. Shen, New J. Phys. 12, 043048 (2010).

${ }^{31}$ B. A. Bernevig and S. C. Zhang, Phys. Rev. Lett. 95, 016801 (2005).

${ }^{32}$ B. Buttner, C. X. Liu, G. Tkachov, E. G. Novik, C. Brune, H. Buhmann, E. M. Hankiewicz, P. Recher, B. Trauzettel, S. C. Zhang, and L. W. Molenkamp, e-print arXiv:1009.2248 (2010).

${ }^{33}$ Y. Hatsugai, Phys. Rev. Lett. 71, 3697 (1993).

${ }^{34}$ D. J. Thouless, M. Kohmoto, M. P. Nightingale, and M. den Nijs, Phys. Rev. Lett. 49, 405 (1982).

${ }^{35}$ M. C. Chang and Q. Niu, J. Phys. Condens. Matter 20, 193202 (2008).

${ }^{36}$ D. Xiao, M. C. Chang, and Q. Niu, Rev. Mod. Phys. 82, 1959 (2010).

${ }^{37}$ M. Buttiker, Phys. Rev. Lett. 57, 1761 (1986).

${ }^{38}$ S. Datta, Electronic Transport in Mesoscopic Systems (Cambridge University Press, Cambridge, 1995).

${ }^{39}$ E. M. Hankiewicz, L. W. Molenkamp, T. Jungwirth, and Jairo Sinova, Phys. Rev B 70, 241301(R) (2004).

${ }^{40}$ J. Li, L. B. Hu, and S. Q. Shen, Phys. Rev. B 71, 241305(R) (2005).

${ }^{41}$ S. Murakami, N. Nagaosa, and S. C. Zhang, Science 301, 1348 (2003).

${ }^{42}$ J. Sinova, D. Culcer, Q. Niu, N. A. Sinitsyn, T. Jungwirth, and A. H. MacDonald, Phys. Rev. Lett. 92, 126603 (2004). 
${ }^{43}$ S. Q. Shen, Phys. Rev. B 70, 081311(R) (2004).

${ }^{44}$ J. Sinova, S. Murakami, S. Q. Shen, and M. S. Choi, Solid State Commun. 138, 214 (2006).

${ }^{45}$ C. L. Kane and E. J. Mele, Phys. Rev. Lett. 95, 226801 (2005).

${ }^{46}$ J. Li, R. L. Chu, J. K. Jain, and S. Q. Shen, Phys. Rev. Lett. 102, 136806 (2009).

${ }^{47}$ C. W. Groth, M. Wimmer, A. R. Akhmerov, J. Tworzydlo, and C. W. J. Beenakker, Phys. Rev. Lett. 103, 196805 (2009).
${ }^{48}$ L. Y. Ma, L. Tang, Z. L. Guan, K. He, K. An, X. C. Ma, J. F. Jia, Q. K. Xue, Y. Han, S. Huang, and F. Liu, Phys. Rev. Lett. 97, 266102 (2006).

${ }^{49}$ T. Zhang, P. Cheng, X. Chen, J. F. Jia, X. C. Ma, K. He, L. L. Wang, H. J. Zhang, X. Dai, Z. Fang, X. C. Xie, and Q. K. Xue, Phys. Rev. Lett. 103, 266803 (2009).

${ }^{50}$ C. Brune, A. Roth, E. G. Novik, M. Konig, H. Buhamann, E. M. Hankeiwicz, W. Hanke, J. Sinova, and L. W. Molenkamp, Nature Phys. 6, 448 (2010). 